\title{
Valuation of Two-Factor Interest Rate Contingent Claims using Green's Theorem
}

\author{
Ghulam Sorwar ${ }^{\mathrm{a}, *}$, Giovanni Barone-Adesi ${ }^{\mathrm{b}, \boldsymbol{\dagger}, *}$ \\ ${ }^{a}$ University of Nottingham, Jubilee Campus, Nottingham, NG8 1BB, UK \\ ${ }^{\mathrm{b}}$ Swiss Finance Institute at the University of Lugano, Via Buffi 13, 6900,Lugano, \\ Switzerland
}

\begin{abstract}
Over the years a number of two-factor interest rate models have been proposed that have formed the basis for the valuation of interest rate contingent claims. This valuation equation often takes the form of a partial differential equation, that is solved using the finite difference approach. In the case of two factor models this has resulted in solving two second order partial derivatives leading to boundary errors, as well as numerous first order derivatives. In this paper we demonstrate that using Green's theorem second order derivatives can be reduced to first order derivatives, that can be easily discretised; consequently two factor partial differential equations are easier to discretise than one factor partial differential equations. We illustrate our approach by applying it to value contingent claims based on the two factor CIR model. We provide numerical examples which illustrates that our approach shows excellent agreement with analytical prices and the popular Crank Nicolson method.
\end{abstract}

KEYWORDS: Box method, derivatives, Green's theorem

\footnotetext{
*Corresponding author. Email: ghulam.sorwar@nottingham.ac.uk; Tel:+44 115951 5489; Fax: +44 115 8466667

† Email: Baroneg@lu.unisi.ch; Tel: +41 58666 4753; Fax: +41 586664334

$\$$ Giovanni Barone-Adesi gratefully acknowledged the financial support of the Swiss National Science Foundation (NCCR FINRISK)
} 


\title{
Valuation of Two-Factor Interest Rate Contingent Claims using Green's Theorem
}

\begin{abstract}
Over the years a number of two-factor interest rate models have been proposed that have formed the basis for the valuation of interest rate contingent claims. This valuation equation often takes the form of a partial differential equation, that is solved using the finite difference approach. In the case of two factor models this has resulted in solving two second order partial derivatives leading to boundary errors, as well as numerous first order derivatives. In this paper we demonstrate that using Green's theorem second order derivatives can be reduced to first order derivatives, that can be easily discretised; consequently two factor partial differential equations are easier to discretise than one factor partial differential equations. We illustrate our approach by applying it to value contingent claims based on the two factor CIR model. We provide numerical examples which illustrates that our approach shows excellent agreement with analytical prices and the popular Crank Nicolson method.
\end{abstract}

KEYWORDS:Box method, Derivatives, Green's Theorem 


\section{Introduction}

The fixed income market is one of the largest sectors of the financial markets where billions of dollars worth of assets are traded daily. Over the years a variety of interest rate models, both single-factor and multi-factors have been proposed that have formed the basis for the valuation of fixed income instruments. The most general of the singlefactor interest rate models is the model proposed by Chan et-al (1992) (henceforth CKLS). The CKLS model encloses many of the earlier single-factor models such as that proposed by Vasicek (1977) and Cox et-al(1985b) (henceforth CIR). The main advantage of one-factor models is their simplicity as the entire yield curve is a function of a single state variable. However, there are several problems associated with single-factor models. First, single factor models assume that changes in the yield curve, and hence bond returns, are perfectly correlated. Secondly, the shape of the yield curve is severely restricted. To overcome these limitations a number of two factor models have been proposed, including those by Brennan and Schwartz (1979), Cox et-al (1985b) amongst others.

Complexity of the underlying stochastic processes used to model fixed income instruments means that except in few specific cases, numerical techniques are necessary. Widely used numerical techniques include the lattice approach of Cox et-al (1979), Monte Carlo Simulation of Boyle (1977) and the finite difference approach. The finite difference approach is widely used both in the financial markets and in academia. This approach involves transforming the valuation equation expressed as a partial differential equation into a set of finite difference equations. This set is then solved either iteratively 
or by elimination to obtain the value of the instruments. Number of researchers including Brennan and Schwartz (1979), Courtadon (1982), Hull and White (1990) have applied the finite difference approach to value fixed income instruments. Sorwar et-al (2007) introduced the Box method from the physical sciences and demonstrated by numerical experimentation that the Box method was superior to the finite difference approach for valuing fixed income instruments. In this paper we further develop the approach of Sorwar et-al (2007) to value two factor fixed income instruments. We demonstrate that by avoiding the traditional route of solving two factor valuation equation as suggested by Brennan and Schwartz (1979) and Hull and White (1990) and others, we in fact end up with a simpler valuation problem where only first order derivatives are present. Our approach involves using Green's theorem to convert a surface integral into a line integral, more specifically it allows us to convert a second order derivative into a first order derivative.

In Section 2, we provide a description of the general problem. We then state the valuation equation based on two-factor interest rate models. We then prove using Green's theorem how the valuation equation can be reduced to a form that involves first order derivatives only. We further demonstrate our approach in depth by applying the proposed technique to the two-factor CIR model. We develop the algorithm in depth and illustrate its accuracy by comparing calculated bond prices with analytical bond prices. In Section 3, we discuss the results. In the final Section we offer conclusions and discuss avenues for future research. 


\section{Two Factor General Diffusion}

\subsection{Deriving simplified equation using Green's theorem}

Assume two stochastic processes that dictate contingent claim prices

$$
\begin{aligned}
& \left.d x=a_{x} \underline{\left(\theta_{x}\right.}, x, t\right) d t+\sigma_{x}(x, t) d z_{x} \\
& \left.d y=a_{y} \underline{\left(\theta_{y}\right.}, y, t\right) d t+\sigma_{y}(y, t) d z_{y}
\end{aligned}
$$

where $x$ and $y$ are model specific stochastic parameters; $\left.a_{x}\left(\underline{\theta_{x}}, x, t\right), a_{y} \underline{\theta_{y}}, y, t\right)$ are the drift terms and $\sigma_{x}(x, t), \sigma_{y}(y, t)$ are the diffusion terms and $\underline{\theta_{x}}$ and $\theta_{y}$ are a vector of model specific parameters.

Using standard hedging arguments and taking instantaneous short rate as $r(x, y)$, the above two stochastic processes lead to the following partial differential equation for contingent claim $U(x, y, \tau)$ at time $t$, expiring at time $T$, and $\tau=T-t$; subject to the usual boundary conditions.

$$
\frac{1}{2} \sigma_{x}^{2} \frac{\partial^{2} U}{\partial x^{2}}+\frac{1}{2} \sigma_{y}^{2} \frac{\partial^{2} U}{\partial y^{2}}+\rho \sigma_{x} \sigma_{y} \frac{\partial^{2} U}{\partial x \partial y}+a_{x} \frac{\partial U}{\partial x}+a_{y} \frac{\partial U}{\partial y}-r(x, y) U=\frac{\partial U}{\partial \tau}
$$

The standard approach in finance literature is to discretise equation (3) using finite difference approximations both for the first order and second order derivatives as in Brennan and Schwartz (1979), Hull and White (1990) etc. Instead of following this traditional route, we generalise the approach of Sorwar et-al (2007) to simplify equation (3): 


$$
\begin{aligned}
& \frac{\partial}{\partial x}\left(S(x, y) \frac{\partial U}{\partial x}\right)+\frac{\partial}{\partial y}\left(W(x, y) \frac{\partial U}{\partial y}\right)+\rho R(x, y) \sigma_{x} \sigma_{y} \frac{\partial^{2} U}{\partial x \partial y} \\
& -R(x, y) r(x, y) U-R(x, y) \frac{\partial U}{\partial \tau}=0
\end{aligned}
$$

PROPOSITION 2.1: Using Green's theorem we can reduce equation (2.4) to the following form:

$$
\begin{aligned}
& \iint_{c_{i j}}\left(S \frac{\partial U}{\partial x} d y-W \frac{\partial U}{\partial y} d x\right)+\iint_{r_{i j}} \rho R(x, y) \sigma_{x} \sigma_{y} \frac{\partial^{2} U}{\partial x \partial y} d x d y \\
& -\iint_{r_{i j}} R(x, y) r(x, y) U d x d y-\iint_{r_{i j}} R(x, y) \frac{\partial U}{\partial \tau} d x d y=0
\end{aligned}
$$

Proof: As in Sorwar et-al (2007) we integrate equation (4) before descretising. Unlike Sorwar et-all (2007) where only a single factor process is considered, consideration of two factors leads to an equation, which involves integrating over a surface $r_{i j}$ :

$$
\begin{aligned}
& \iint_{r_{i j}} \frac{\partial}{\partial x}\left(S(x, y) \frac{\partial U}{\partial x}\right) d x d y+\iint_{r_{i j}} \frac{\partial}{\partial y}\left(W(x, y) \frac{\partial U}{\partial y}\right) d x d y \\
& -\iint_{r_{i j}} R(x, y) r(x, y) U d x d y-\iint_{r_{i j}} R(x, y) \frac{\partial U}{\partial \tau} d x d y=0
\end{aligned}
$$

From Green's theorem, we know that for any two differentiable functions $A(x, y)$ and $B(x, y)$ defined in $r_{i j}$ :

$$
\iint_{r_{i j}}\left(\frac{\partial A}{\partial x}-\frac{\partial B}{\partial y}\right) d x d y=\oint_{c_{i j}}(B d x+A d y)
$$

where $c_{i j}$ is the boundary of $r_{i j}$ and the line integral is taken in the positive sense.

Rewriting equation (7) by letting:

$$
\begin{aligned}
& A=S \frac{\partial U}{\partial x} \\
& B=-W \frac{\partial U}{\partial y}
\end{aligned}
$$




$$
\iint_{r_{i j}}\left(\frac{\partial A}{\partial x}-\frac{\partial B}{\partial y}\right) d x d y=\int_{c_{i j}}\left(S \frac{\partial U}{\partial x} d y-W \frac{\partial U}{\partial y} d x\right)
$$

Substituting the right hand side of equation (10) for the first two left hand side terms of equation (6) yields the required equation.

\subsection{Application to two factor CIR processes}

To illustrate our approach, we concentrate on two-factor model of the term structure, set within the CIR framework (1985a, 1985b). Two dependent state variables $x$ and $y$ determine the nominal instantaneous interest rate $r(x, y)$ :

$$
r(x, y)=x+y
$$

We assume the risk-adjusted factors are generated by independent square root processes as below:

$$
\begin{aligned}
& d x=\left(\kappa_{1} \theta_{1}-\kappa_{1} x-\lambda_{1} x\right) d t+\sigma_{1} \sqrt{x} d z_{x} \\
& d y=\left(\kappa_{2} \theta_{2}-\kappa_{2} y-\lambda_{2} y\right) d t+\sigma_{2} \sqrt{y} d z_{y}
\end{aligned}
$$

The valuation equation for contingent claims $U(x, y, \tau)$ assuming no intermediate cash flows is:

$$
\begin{aligned}
& \frac{1}{2} \sigma_{1}^{2} x \frac{\partial^{2} U}{\partial x^{2}}+\frac{1}{2} \sigma_{2}^{2} y \frac{\partial^{2} U}{\partial y^{2}}+\rho \sigma_{1} \sigma_{2} \sqrt{x y} \frac{\partial^{2} U}{\partial x \partial y} \\
& +\left[\kappa_{1} \theta_{1}-x\left(\kappa_{1}-\lambda_{1}\right)\right] \frac{\partial U}{\partial x}+\left[\kappa_{2} \theta_{2}-x\left(\kappa_{2}-\lambda_{2}\right)\right] \frac{\partial U}{\partial y}-(x+y) U=\frac{\partial U}{\partial \tau}
\end{aligned}
$$


Dividing equation (14) by $x y$ gives:

$$
\begin{aligned}
& \frac{1}{2} \frac{\sigma_{1}^{2}}{y} \frac{\partial^{2} U}{\partial x^{2}}+\frac{1}{2} \frac{\sigma_{2}^{2}}{x} \frac{\partial^{2} U}{\partial y^{2}}+\frac{\rho \sigma_{1} \sigma_{2}}{\sqrt{x y}} \frac{\partial^{2} U}{\partial x \partial y} \\
& +\left[\frac{\kappa_{1} \theta_{1}}{x y}-\frac{1}{y}\left(\kappa_{1}-\lambda_{1}\right)\right] \frac{\partial U}{\partial x}+\left[\frac{\kappa_{2} \theta_{2}}{x y}-\frac{1}{x}\left(\kappa_{2}-\lambda_{2}\right)\right] \frac{\partial U}{\partial y}-\left(\frac{1}{y}+\frac{1}{x}\right) U=\frac{1}{x y} \frac{\partial U}{\partial \tau}
\end{aligned}
$$

Now consider $x$ and $y$ derivatives of equation (15) separately in terms of functions $P(x)$ and $Q(y)$ respectively:

$$
\begin{aligned}
& \frac{1}{2} \frac{\sigma_{1}^{2}}{y} \frac{\partial^{2} U}{\partial x^{2}}+\left[\frac{\kappa_{1} \theta_{1}}{x y}-\frac{1}{y}\left(\kappa_{1}-\lambda_{1}\right)\right] \frac{\partial U}{\partial x}=\frac{1}{P(x)} \frac{\partial}{\partial x}\left(P(x) \frac{\partial U}{\partial x}\right) \\
& \frac{1}{2} \frac{\sigma_{2}^{2}}{x} \frac{\partial^{2} U}{\partial y^{2}}+\left[\frac{\kappa_{2} \theta_{2}}{x y}-\frac{1}{x}\left(\kappa_{2}-\lambda_{2}\right)\right] \frac{\partial U}{\partial y}=\frac{1}{Q(y)} \frac{\partial}{\partial y}\left(P(y) \frac{\partial U}{\partial y}\right)
\end{aligned}
$$

Expanding equations (16) and (17) and integrating gives:

$$
\begin{aligned}
& P(x)=\exp \left\{\frac{2 \kappa_{1} \theta_{1}}{\sigma_{1}^{2}} \ln (x)-\frac{2}{\sigma_{1}^{2}}\left(\kappa_{1}-\lambda_{1}\right) x\right\}=x^{\frac{2 \kappa_{1} \theta_{1}}{\sigma_{1}^{2}}} \exp \left\{-\frac{2}{\sigma_{1}^{2}}\left(\kappa_{1}-\lambda_{1}\right) x\right\} \\
& Q(y)=\exp \left\{\frac{2 \kappa_{2} \theta_{2}}{\sigma_{2}^{2}} \ln (y)-\frac{2}{\sigma_{2}^{2}}\left(\kappa_{2}-\lambda_{2}\right) y\right\}=y^{\frac{2 \kappa_{2} \theta_{2}}{\sigma_{2}^{2}}} \exp \left\{-\frac{2}{\sigma_{2}^{2}}\left(\kappa_{2}-\lambda_{2}\right) y\right\}
\end{aligned}
$$

We now define a new function $R(x, y)$ which is a product of $P(x)$ and $Q(y)$ :

$$
R(x, y)=x^{\alpha_{0}} y^{\beta_{0}} \exp \left(-\alpha_{1} x-\beta_{1} y\right)
$$


where:

$$
\alpha_{0}=\frac{2 \kappa_{1} \theta_{1}}{\sigma_{1}^{2}}, \beta_{0}=\frac{2 \kappa_{2} \theta_{2}}{\sigma_{2}^{2}}, \alpha_{1}=\frac{2}{\sigma_{1}^{2}}\left(\kappa_{1}-\lambda_{1}\right), \beta_{1}=\frac{2}{\sigma_{2}^{2}}\left(\kappa_{2}-\lambda_{2}\right)
$$

Further define:

$$
\begin{aligned}
& S(x, y)=\frac{\sigma_{1}^{2}}{2 y} R(x, y) \\
& W(x, y)=\frac{\sigma_{2}^{2}}{2 x} R(x, y)
\end{aligned}
$$

Thus the original partial differential equation (14) becomes:

$$
\frac{\partial}{\partial x}\left(S \frac{\partial U}{\partial x}\right)+\frac{\partial}{\partial y}\left(W \frac{\partial U}{\partial y}\right)+\frac{\rho \sigma_{1} \sigma_{2}}{\sqrt{x y}} R \frac{\partial^{2} U}{\partial x \partial y}-R\left(\frac{1}{x}+\frac{1}{y}\right) U=\frac{R}{x y} \frac{\partial U}{\partial \tau}
$$

Taking the forward Euler-difference for the time derivative in equation (3.13) gives us:

$$
\frac{\partial U}{\partial \tau}=\frac{U-U_{0}}{\Delta t}
$$

Substituting the above approximation and re-arranging equation (23) yields:

$$
-\left[\frac{\partial}{\partial x}\left(S \frac{\partial U}{\partial x}\right)+\frac{\partial}{\partial y}\left(W \frac{\partial U}{\partial y}\right)\right]-\frac{\rho \sigma_{1} \sigma_{2}}{\sqrt{x y}} R \frac{\partial^{2} U}{\partial x \partial y}-R\left(\frac{1}{x}+\frac{1}{y}+\frac{1}{x y \Delta t}\right) U=\frac{R U_{0}}{x y \Delta t}
$$

Using Proposition (2.1) allows us to transform equation (25) to the following form involving a line integral:

$-\oint_{c_{i j}}\left(S \frac{\partial U}{\partial x} d y-W \frac{\partial U}{\partial y} d x\right)-\iint_{r_{i j}} R \frac{\rho \sigma_{1} \sigma_{2}}{\sqrt{x y}} \frac{\partial^{2} U}{\partial x \partial y} d x d y+\iint_{r_{i j}} R\left(\frac{1}{x}+\frac{1}{y}+\frac{1}{x y \Delta t}\right) U d x d y=\iint_{r_{i j}} \frac{R}{x y \Delta t} U_{0} d x d y$ 
For the line integral noting that $d y$ is zero when moving along the $x$ direction and $d x$ is zero when moving along the $y$ direction we have:

$$
-\oint_{c_{i j}}\left(S \frac{\partial U}{\partial x} d y-W \frac{\partial U}{\partial y} d x\right)=\int_{1}^{2} W \frac{\partial C}{\partial y} d x-\int_{2}^{3} S \frac{\partial C}{\partial x} d y+\int_{3}^{4} W \frac{\partial C}{\partial y} d x-\int_{4}^{1} S \frac{\partial C}{\partial x} d y
$$

We let $x, y$ and $\tau$ take value on the interval $\Gamma_{x}=[0, X], \Gamma_{y}=[0, Y], \mathrm{T}=[0, T]$. To solve the above equation we need to fit the space $\Gamma_{x} \times \Gamma_{y} \times \mathrm{T}$. We let $\Delta x, \Delta y$ and $\Delta t$ represent the grid spacing the $x, y$ and $\tau$ direction respectively, such that:

$x_{i}=i \Delta x$ for $0 \leq N_{x}$ such that $\Gamma_{x}=N_{x} \Delta x$

$y_{j}=j \Delta y \quad$ for $0 \leq N_{y}$ such that $\Gamma_{y}=N_{y} \Delta y$

$\tau_{m}=m \Delta t$ for $0 \leq M$ such that $\mathrm{T}=M \Delta t$

Further we take:

$$
\begin{aligned}
& x_{i+\frac{1}{2}}=X_{U}=\frac{x_{i+1}+x_{i}}{2} \\
& x_{i-\frac{1}{2}}=X_{L}=\frac{x_{i}+x_{i-1}}{2} \\
& y_{j+\frac{1}{2}}=Y_{U}=\frac{y_{j+1}+y_{j}}{2} \\
& y_{j-\frac{1}{2}}=Y_{L}=\frac{y_{j}+y_{j-1}}{2}
\end{aligned}
$$

Discretising each of the line integrals and simplifying gives:

$$
\begin{aligned}
-\oint_{c_{i j}}\left(S \frac{\partial U}{\partial x} d y-W \frac{\partial U}{\partial y} d x\right) & =\frac{\Delta x}{\Delta y}\left[W_{i, j+\frac{1}{2}}\left(U_{i j}^{m}-U_{i, j+1}^{m}\right)+W_{i, j-\frac{1}{2}}\left(U_{i j}^{m}-U_{i, j-1}^{m}\right)\right] \\
& +\frac{\Delta y}{\Delta x}\left[S_{i+\frac{1}{2}, j}\left(U_{i j}^{m}-U_{i+1, j}^{m}\right)+S_{i-\frac{1}{2}, j}\left(U_{i j}^{m}-U_{i-1, j}^{m}\right)\right]
\end{aligned}
$$


Discretising the remaining components gives

$$
\begin{gathered}
\iint_{r_{i j}} \frac{R}{x y \Delta t} U_{0} d x d y \approx \frac{R_{i j} U_{i j}^{m-1}}{\Delta t} \int_{X_{L}}^{X_{U}} \int_{Y_{L}}^{Y_{U}} \frac{1}{x y} d x d y=\frac{R_{i j}}{\Delta t} \ln \left(\frac{X_{U} Y_{U}}{X_{L} Y_{L}}\right) U_{i j}^{m-1}=H_{i j} U_{i j}^{m-1} \\
\iint_{r_{i j}} R\left(\frac{1}{x}+\frac{1}{y}+\frac{1}{x y \Delta t}\right) U d x d y=\int_{X_{L}}^{X_{Y_{L}}} \int_{Y_{U}} R\left(\frac{1}{x}+\frac{1}{y}+\frac{1}{x y \Delta t}\right) U d x d y \\
\approx R_{i j}\left[\ln \left(\frac{Y_{U}}{Y_{L}}\right)\left(X_{U}-X_{L}\right)+\ln \left(\frac{X_{U}}{X_{L}}\right)\left(Y_{U}-Y_{L}\right)+\frac{1}{\Delta t} \ln \left(\frac{X_{U} Y_{U}}{X_{L} Y_{L}}\right)\right] U_{i j}^{m}=G_{i j} U_{i j}^{m} \\
-\iint_{r_{i j}} R \frac{\rho \sigma_{1} \sigma_{2}}{\sqrt{x y} \frac{\partial^{2} U}{\partial x \partial y} d x d y=}-\frac{\rho \sigma_{1} \sigma_{2}}{16 \Delta x \Delta y}\left(\sqrt{X_{U}}-\sqrt{X_{L}}\right)\left(\sqrt{Y_{U}}-\sqrt{Y_{L}}\right) \times \\
\left(U_{i+1, j+1}^{m}-U_{i+1, j-1}^{m}-U_{i-1, j+1}^{m}+U_{i-1, j-1}^{m}\right)
\end{gathered}
$$

Collecting all the terms and re-arranging gives the final matrix equation as:

$$
\begin{aligned}
& A_{i j} U_{i j}^{m}+C_{i j} U_{i, j+1}^{m}+D_{i j} U_{i, j-1}^{m}+E_{i j} U_{i+1, j}^{m}+F_{i j} U_{i-1, j}^{m} \\
& +I_{i j}\left(U_{i+1, j+1}^{m}-U_{i+1, j-1}^{m}-U_{i-1, j+1}^{m}+U_{i-1, j-1}^{m}\right)=H_{i j} U_{i j}^{m-1} \\
& \text { where } \\
& A_{i j}=\frac{\Delta x}{\Delta y}\left(\frac{W_{i, j+\frac{1}{2}}}{R_{i j}}+\frac{W_{i, j-\frac{1}{2}}}{R_{i j}}\right)+\frac{\Delta y}{\Delta x}\left(\frac{S_{i+\frac{1}{2}, j}}{R_{i j}}+\frac{S_{i-\frac{1}{2}, j}}{R_{i j}}\right)+G_{i j} \\
& C_{i j}=-\frac{\Delta x}{\Delta y} \frac{W_{i, j+\frac{1}{2}}}{R_{i j}}, D_{i j}=-\frac{\Delta x}{\Delta y} \frac{W_{i, j-\frac{1}{2}}}{R_{i j}} \\
& E_{i j}=-\frac{\Delta y}{\Delta x} \frac{S_{i+\frac{1}{2}, j}}{R_{i j}}, F_{i j}=-\frac{\Delta y}{\Delta x} \frac{S_{i-\frac{1}{2}, j}}{R_{i j}} \\
& I_{i j}=-\frac{\rho \sigma_{1} \sigma_{2}}{16 \Delta x \Delta y}\left(\sqrt{X_{U}}-\sqrt{X}\right)\left(\sqrt{Y_{U}}-\sqrt{Y_{L}}\right)
\end{aligned}
$$


To determine contingent claim prices we use the following SOR iteration subject to appropriate boundary conditions:

$$
\begin{aligned}
& Z_{i j}^{m}=\frac{1}{A_{i j}}\left(\begin{array}{l}
G_{i j} U_{i j}^{m-1}-C_{i j} U_{i, j+1}^{m}-D_{i j} U_{i, j+1}^{m}-E_{i j} U_{i+1, j}^{m}-F_{i j} U_{i-1, j}^{m} \\
-I_{i j}\left(U_{i+1, j+1}^{m}-U_{i+1, j-1}^{m}-U_{i-1, j+1}^{m}+U_{i-1, j-1}^{m}\right.
\end{array}\right) \\
& U_{i j}^{m}=\omega Z_{i j}^{m}+(1-\omega) U_{i j}^{m-1} \\
& \text { for } i=1, \ldots \ldots . ., N_{x}-1 \quad j=1, \ldots \ldots . ., N_{y}-1 \text { and } \omega \in(1,2]
\end{aligned}
$$

\section{Discussion of Results}

In Table 1 and Table 2, we compare numerically evaluated bond prices with analytical bond prices. The maturities of the bonds range from 1 year to 15 years. The face value of the zero coupon bonds are $\$ 100$. Interest rates ranging from 5\% to $9 \%$ are considered. For direct comparison with analytical bond prices the correlation coefficient is zero. Further we alter the annual number of time steps from 20 to 50. This variation serves as a check on the stability of the numerical scheme.

In Table 3 to Table 6 we compare American call and put prices calculated using the Box Method and the Crank-Nicolson method based on time step of 1/50 per year. In Table 3 to Table 4 we focus on short expiry options, whereas in Table 5 - Table 6 we focus on long dated options.

Examining Table 1 and Table 2, we can conclude the following. First, it is possible to obtain accurate bond prices using the Box Method and Crank Nicolson, with as little as 20 annual time steps per year. For example, for a five year bond, with $x=5 \%, y=5 \%$ the analytical bond price is $\$ 59.6215$ compared with the Box price of $\$ 59.5986$ and Crank Nicolson price of $\$ 59.3703$. Secondly, increasing the annual number of time steps 
leads to more accurate bond prices. Again, considering the same five year bond at the same interest rates, we find the Box price with 50 annual time steps is $\$ 59.5396$ and Crank Nicolson price is $\$ 59.3710$. Thirdly, we find that Crank Nicolson leads to more accurate bond prices for short term bonds, whereas the Box Method leads to more accurate bond prices at longer maturities. Finally, bond prices show expected trends. Thus, as the short-term interest rates and the term to maturity of the bonds increase, bond prices decline. From Table 3 - Table 6 we find that Box call options are always higher than Crank Nicoslon call prices and Box put are always lower than Crank Nicolson puts. This discrepancy is larger for longer maturity options. Given the evidence in Table Table 2, it is likely that the Crank-Nicolson method is marginally less accurate than the Box method for longer expiry options.

\section{Conclusion}

This paper focuses on the valuation of two factor interest rate, contingent claims. By expanding the earlier work of Sorwar et-al (2007), this paper proposes a more elegant technique based on Green's theorem to solve the valuation equation.

Further this paper illustrates how the proposed technique can be applied in the case of a specific two-factor model. Concentrating on the two factor CIR model, this paper illustrates the steps necessary to develop the system of equations needed to value the contingent claims. Numerical experimentation shows excellent agreement between analytical CIR bond prices and computed CIR bond prices. 
Over the years a large number of two factor interest rate models have been proposed, many of which do not lead to analytical contingent claim prices. The proposed Box method offers an easy and quick route to examine the pricing implications of these models. These implications remain the avenue of future research. 
Table 1. Bond Prices calculated analytically (CIR), using the Box and the Crank Nicholson methods.

\begin{tabular}{|c|c|c|c|c|c|c|c|c|c|c|}
\hline \multicolumn{11}{|c|}{$\kappa_{1}=0.5, \sigma_{1}=0.15, \theta_{1}=0.06, \lambda_{1}=0$} \\
\hline \multicolumn{11}{|c|}{$\kappa_{2}=0.005, \sigma_{2}=0.07, \theta_{2}=0.03, \lambda_{2}=0$} \\
\hline \multicolumn{11}{|c|}{$\rho=0, \Delta \mathrm{r}=0.5 \%, \Delta \mathrm{t}=1 / 20$ years } \\
\hline Maturity & Method & $x=5, y=5$ & $x=5, y=7$ & $x=5, y=9$ & $x=7, y=5$ & $x=7, y=7$ & $x=7, y=9$ & $x=9, y=5$ & $x=9, y=7$ & $x=9, y=9$ \\
\hline \multirow[t]{3}{*}{1 year } & Anal. & 90.3114 & 88.5290 & 86.7817 & 88.9052 & 87.1506 & 85.4305 & 87.5209 & 85.7936 & 84.1003 \\
\hline & Box & 90.3401 & 88.5663 & 86.8295 & 88.9503 & 87.2048 & 85.4958 & 87.5837 & 85.8662 & 84.1844 \\
\hline & $\mathrm{CN}$ & 90.3115 & 88.5291 & 86.7818 & 88.9052 & 87.1505 & 85.4304 & 87.5208 & 85.7934 & 84.1001 \\
\hline \multirow[t]{3}{*}{5 years } & Anal. & 59.4534 & 53.9762 & 48.9872 & 57.3677 & 52.0739 & 47.2687 & 55.3551 & 50.2471 & 45.6105 \\
\hline & Box & 59.5986 & 54.1285 & 49.1643 & 57.5013 & 52.2256 & 47.4366 & 55.4824 & 50.3937 & 45.7734 \\
\hline & $\mathrm{CN}$ & 59.3703 & 53.8936 & 48.9214 & 57.3190 & 52.0316 & 47.2310 & 55.3232 & 50.2200 & 45.5865 \\
\hline \multirow[t]{3}{*}{10 years } & Anal. & 35.9733 & 30.0203 & 25.0524 & 34.6260 & 28.8960 & 24.1142 & 33.3292 & 27.8138 & 23.2110 \\
\hline & Box & 36.0449 & 30.1646 & 25.2158 & 34.6289 & 29.0271 & 24.2657 & 33.3754 & 27.9348 & 23.3533 \\
\hline & $\mathrm{CN}$ & 35.5625 & 29.6589 & 24.7454 & 34.3248 & 28.6302 & 23.8882 & 33.0924 & 27.6044 & 23.0330 \\
\hline \multirow[t]{3}{*}{ 15years } & Anal. & 22.6975 & 17.7325 & 13.8535 & 21.8439 & 17.0656 & 13.3325 & 21.0224 & 16.4238 & 12.8311 \\
\hline & Box & 22.4823 & 17.7154 & 13.9088 & 21.6825 & 17.0444 & 13.3826 & 20.8088 & 16.4000 & 12.8772 \\
\hline & $\mathrm{CN}$ & 22.0139 & 17.1259 & 13.3483 & 21.3032 & 16.5823 & 12.9285 & 20.5708 & 16.0177 & 12.4908 \\
\hline
\end{tabular}


Table 2. Bond Prices calculated analytically (CIR), using the Box and the Crank Nicholson methods.

\begin{tabular}{|c|c|c|c|c|c|c|c|c|c|c|}
\hline \multicolumn{11}{|c|}{$\kappa_{1}=0.5, \sigma_{1}=0.15, \theta_{1}=0.06, \lambda_{1}=0$} \\
\hline \multicolumn{11}{|c|}{$\kappa_{2}=0.005, \sigma_{2}=0.07, \theta_{2}=0.03, \lambda_{2}=0$} \\
\hline \multicolumn{11}{|c|}{$\rho=0, \Delta \mathrm{r}=0.5 \%, \Delta \mathrm{t}=1 / 50$ years } \\
\hline Maturity & Method & $x=5, y=5$ & $x=5, y=7$ & $x=5, y=9$ & $x=7, y=5$ & $x=7, y=7$ & $x=7, y=9$ & $x=9, y=5$ & $x=9, \mathrm{y}=7$ & $x=9, y=9$ \\
\hline \multirow[t]{3}{*}{1 year } & Anal. & 90.3114 & 88.5290 & 86.7817 & 88.9052 & 87.1506 & 85.4305 & 87.5209 & 85.7936 & 84.1003 \\
\hline & Box & 90.3284 & 88.5485 & 86.8050 & 88.9272 & 87.1756 & 85.4595 & 87.5493 & 85.8254 & 84.1364 \\
\hline & $\mathrm{CN}$ & 90.3114 & 88.5290 & 86.7817 & 88.9052 & 87.1505 & 85.4305 & 87.5209 & 85.7936 & 84.1003 \\
\hline \multirow[t]{3}{*}{5 years } & Anal. & 59.4534 & 53.9762 & 48.9872 & 57.3677 & 52.0739 & 47.2687 & 55.3551 & 50.2471 & 45.6105 \\
\hline & Box & 59.5396 & 54.0588 & 49.0817 & 57.4402 & 52.1545 & 47.3534 & 55.4192 & 50.3212 & 45.6895 \\
\hline & $\mathrm{CN}$ & 59.3710 & 53.8926 & 48.9199 & 57.3201 & 52.0311 & 47.2301 & 55.3246 & 50.2198 & 45.5859 \\
\hline \multirow[t]{3}{*}{10 years } & Anal. & 35.9733 & 30.0203 & 25.0524 & 34.6260 & 28.8960 & 24.1142 & 33.3292 & 27.8138 & 23.2110 \\
\hline & Box & 35.9581 & 30.0844 & 25.1361 & 34.5994 & 28.9500 & 24.1891 & 33.2950 & 27.8606 & 23.2796 \\
\hline & $\mathrm{CN}$ & 35.5671 & 29.6598 & 24.7447 & 34.3294 & 28.6314 & 23.8878 & 33.0970 & 27.6057 & 23.0328 \\
\hline \multirow[t]{3}{*}{15 years } & Anal. & 22.6975 & 17.7325 & 13.8535 & 21.8439 & 17.0656 & 13.3325 & 21.0224 & 16.4238 & 12.8311 \\
\hline & Box & 22.3896 & 17.6430 & 13.8477 & 21.5396 & 16.9749 & 13.3240 & 20.7235 & 16.3333 & 12.8210 \\
\hline & $\mathrm{CN}$ & 22.0197 & 17.1284 & 13.3489 & 21.3090 & 16.5848 & 12.9293 & 20.5765 & 16.0203 & 12.4917 \\
\hline
\end{tabular}


Table 3. Option prices calculated using the Box and the Crank Nicholson methods.

\begin{tabular}{|c|c|c|c|c|c|c|}
\hline & & $5, \sigma_{1}=($ & $5, \theta_{1}=$ & 0.06 & & \\
\hline & & $05, \sigma_{2}=$ & $0.07, \theta_{2}$ & 0.0 & $=0$ & \\
\hline & & $\Delta r=0$ & $\%, \Delta \mathrm{t}=$ & $1 / 50$ & & \\
\hline & & & $y=5 \%$ & & & \\
\hline & & aturity $b$ & & $5 y$ & naturity & ond \\
\hline & & expiry & ption & $1 \mathrm{y}$ & xpiry op & ion \\
\hline Method & $\mathrm{K}$ & Call & Put & $\mathrm{K}$ & Call & Put \\
\hline Box & 85 & 9.5109 & 0.0000 & 55 & 9.8923 & 0.0998 \\
\hline $\mathrm{CN}$ & & 9.5004 & 0.0000 & & 9.7339 & 0.0973 \\
\hline Box & 86 & 8.5601 & 0.0000 & 56 & 9.0093 & 0.1669 \\
\hline $\mathrm{CN}$ & & 8.5497 & 0.0000 & & 8.8520 & 0.1655 \\
\hline Box & 87 & 7.6093 & 0.0002 & 57 & 8.1367 & 0.2738 \\
\hline $\mathrm{CN}$ & & 7.5990 & 0.0000 & & 7.9810 & 0.2756 \\
\hline Box & 88 & 6.6585 & 0.0013 & 58 & 7.2786 & 0.4405 \\
\hline $\mathrm{CN}$ & & 6.6483 & 0.0004 & & 7.1253 & 0.4495 \\
\hline Box & 89 & 5.7077 & 0.0107 & 59 & 6.4404 & 0.6953 \\
\hline $\mathrm{CN}$ & & 5.6796 & 0.0055 & & 6.2904 & 0.7185 \\
\hline Box & 90 & 4.7569 & 0.0885 & 60 & 5.6284 & 1.0769 \\
\hline $\mathrm{CN}$ & & 4.7469 & 0.0702 & & 5.4828 & 1.1259 \\
\hline Box & 91 & 3.8065 & 0.6716 & 61 & 4.8500 & 1.6376 \\
\hline $\mathrm{CN}$ & & 3.7964 & 0.6886 & & 4.7100 & 1.7284 \\
\hline Box & 92 & 2.8582 & 1.6716 & 62 & 4.1133 & 2.4604 \\
\hline $\mathrm{CN}$ & & 2.8482 & 1.6886 & & 3.9802 & 2.6290 \\
\hline Box & 93 & 1.9236 & 2.6716 & 63 & 3.4266 & 3.4604 \\
\hline $\mathrm{CN}$ & & 1.9142 & 2.6886 & & 3.3019 & 3.6290 \\
\hline Box & 94 & 1.0537 & 3.8716 & 64 & 2.7980 & 4.4604 \\
\hline $\mathrm{CN}$ & & 1.0478 & 3.6886 & & 2.6826 & 4.6290 \\
\hline Box & 95 & 0.3868 & 4.6716 & 65 & 2.2342 & 5.4604 \\
\hline $\mathrm{CN}$ & & 0.3863 & 4.6886 & & 2.1291 & 5.6290 \\
\hline
\end{tabular}


Table 4. Option prices calculated using the Box and the Crank Nicholson methods.

\begin{tabular}{|c|c|c|c|c|c|c|}
\hline & & $.5, \sigma_{1}=0$ & $15, \theta_{1}=$ & 0.06 & & \\
\hline & & $005, \sigma_{2}=$ & $0.07, \theta_{2}=$ & 0.0 & $=0$ & \\
\hline & & $0, \Delta r=0$ & $\%, \Delta \mathrm{t}=$ & $1 / 50$ & & \\
\hline & & & $=y=5 \%$ & & & \\
\hline & 10 & naturity $b$ & ond & 15 & maturity & ond \\
\hline & $5 y$ & xpiry opti & & 10 & expiry op & ion \\
\hline Method & $\mathrm{K}$ & Call & Put & $\mathrm{K}$ & Call & Put \\
\hline Box & 30 & 18.0992 & 0.1724 & 17 & 16.2769 & 0.2008 \\
\hline $\mathrm{CN}$ & & 17.7206 & 0.1871 & & 15.7250 & 0.2376 \\
\hline Box & 31 & 17.5051 & 0.2495 & 18 & 15.9174 & 0.2940 \\
\hline $\mathrm{CN}$ & & 17.1276 & 0.2720 & & 15.3603 & 0.3479 \\
\hline Box & 32 & 16.9114 & 0.3560 & 19 & 15.5580 & 0.4222 \\
\hline $\mathrm{CN}$ & & 16.5351 & 0.3900 & & 14.9968 & 0.4989 \\
\hline Box & 33 & 16.3184 & 0.5015 & 20 & 15.1986 & 0.5961 \\
\hline $\mathrm{CN}$ & & 15.9434 & 0.5512 & & 14.6345 & 0.7019 \\
\hline Box & 34 & 15.7261 & 0.6978 & 21 & 14.8393 & 0.8287 \\
\hline $\mathrm{CN}$ & & 15.3525 & 0.7694 & & 14.2733 & 0.9708 \\
\hline Box & 35 & 15.1348 & 0.9596 & 22 & 14.4800 & 1.1366 \\
\hline $\mathrm{CN}$ & & 14.7626 & 1.0610 & & 13.9131 & 1.3216 \\
\hline Box & 36 & 14.5447 & 1.3052 & 23 & 14.1209 & 1.5400 \\
\hline $\mathrm{CN}$ & & 14.1741 & 1.4461 & & 13.5538 & 1.7734 \\
\hline Box & 37 & 13.9561 & 1.7564 & 24 & 13.7619 & 2.0636 \\
\hline $\mathrm{CN}$ & & 13.5872 & 1.9481 & & 13.1954 & 2.3470 \\
\hline Box & 38 & 13.3694 & 2.3407 & 25 & 13.4031 & 2.7386 \\
\hline $\mathrm{CN}$ & & 13.0023 & 2.5972 & & 12.8379 & 3.0679 \\
\hline Box & 39 & 12.7849 & 3.0824 & 26 & 13.0446 & 3.6104 \\
\hline $\mathrm{CN}$ & & 12.4198 & 3.4329 & & 12.4811 & 3.9803 \\
\hline Box & 40 & 12.2031 & 4.0419 & 27 & 12.6863 & 4.6104 \\
\hline $\mathrm{CN}$ & & 11.8402 & 4.4329 & & 12.1250 & 4.9803 \\
\hline
\end{tabular}


Reference

Sorwar, G., Barone-Adesi, G., and W. Allegretto (1997): "Valuation of derivatives based on single-factor interest rate models," Global Finance Journal. 18, 251-269.

Boyle, P. P., (1977): “Options: A Monte Carlo Approach,” Journal Financial

Economics. 4, 323-338.

Brennan, M., and E. Schwartz (1979): “A Continuous-Time Approach to the Pricing of Bonds," Journal of Banking Finance 3, 133-155.

Chan, K. C., Karolyi, G. A., Longstaff, F. A., and A.B. Sanders, (1992). “An empirical comparison of the short-term interest rate," Journal of Finance, 1(7), 1209-1227.

Chen, R.R., and L. Scott (1992): "Pricing Interest Rate Option in a Two-Factor CoxIngersoll-Ross Model of the Term Structure," Review of. Financial Studies, 5, 613-636. Courtadon, G., (1982): “The Pricing of Options on Default-Free Bonds,” Journal of Financial and Quantitative Analysis, 17, 75-100.

Cox, J.C., S. A. Ross, and M. Rubinstein (1979): “Option Pricing: A Simple Approach,” Journal of Financial Economics, 7, 229-263.

Cox, J. C, J. E. Ingersoll, and S. A. Ross (1985a): “An Intertemporal General Equilibrium Model of Asset Prices," Econometrica, 53, 363-384.

Cox, J. C, J. E. Ingersoll, and S. A. Ross (1985b): “A Theory of the Term Structure of Interest Rate ," Econometrica, 53, 385-407.

Hull, J., and A. White (1990): "Valuing Derivative Securities Using the Explicit Finite Difference Method," Journal of Financial and Quantitative Analysis 25, 87-99. 
Vasicek, O. A., (1977): “An Equilibrium Characterization of the Term Structure,” Journal of Financial Economics. 5, 177-188. 